\title{
Construction artisanale de turbines hydrauliques pour micro-centrales
}

\author{
Prof. Ing. Zoltán Hosszúréty, Dr. Sc. \\ chaire d'Énergétique thermique \\ Faculté de Métallurgie \\ Université technique de Kosice
}

Pour les micro-turbines, la première exigence est la simplicité de leur construction; on peut, sous certaines conditions, l'accroitre graduellement jusqu'à en permettre la réalisation par un artisan ou même un amateur.

Il n'y a pas lieu de considérer cette tendance comme allant à l'encontre du progrès, du fait que l'on désire alors les construire à l'unité, et non plus en grande série. L'effort correspondant vise à adapter les techniques de fabrication aux conditions sociales et économiques, de manière à en permettre une réalisation rapide. Les particuliers aux revenus modestes, qui bâtissent eux-mêmes leur maison, peuvent également construire leurs micro-centrales par leurs propres moyens, même avec l'aide des subventions de l'État. Il s'agit done de satisfaire aux besoins d'une couche de la population rurale qui, dans beaucoup de pays, en représente une grande partie, et pourrait dans une large mesure utiliser l'énergie hydraulique pour ses besoins domestiques.

La construction dans un petit atelier équipé des machines adéquates peut s'avérer nécessaire, lorsque les paramètres locaux sont en dehors de la zone Q-H assurée par les turbines fabriquées en série industrielle. Ce domaine est représenté, pour la Tchécoslovaquie, par la figure 1 (d'après [1]) et par la figure 2 selon [2].

D'autre part, une extrême simplification de la construction des turbines est appréciable même dans la production industrielle, parce qu'elle abaisse le prix de revient, ce qui est aussi un moyen de contribuer au développement des micro-centrales à usage domestique.

L'auteur a cherché à atteindre ce but au moyen des impératifs suivants relatifs aux moyens de fabrication :

- lors du projet de la turbine, prohiber les pièces moulées ou embouties ; utiliser des matériaux laminés, en excluant tout usinage ;

- exclure par conséquent les tôles gauchies pour la fabrication des aubes et autres parties de la machine ; n'adopter que des formes géométriques réalisables par découpage et pliage, et ne nécessitant pas d'outillage spécial;

- adopter pour les conduits des formes aussi simples que possible, dont la section, tant longitudinale que transversale, soit carrée, rectangulaire ou trapézoïdale ;

- réduire au minimum le nombre et la taille des surfaces usinées, de pièces ajustées l'une à l'autre, et par suite la précision nécessaire au cours de la fabrication ;

- dans le choix du tracé hydraulique, adopter une solution aussi proche que possible de l'isobare, pour rendre très simple ou même inutile l'étanchéité des jeux à l'entrée

- simplifier la technologie de la fabrication, pour que le façonnage se limite à un tournage ;

- effectuer les assemblages par vis et soudure, les points de soudure étant facilement accessibles et réalisables ;

- choisir une vitesse de rotation élevée, afin de réduire le poids ;

- éviter si possible une transmission entre la turbine et le générateur.

Il s'agit non seulement d'en rendre la construction plus simple, mais aussi de la rendre réalisable par des ateliers non spécialisés, ainsi que d'en simplifier la documentation technique, afin que le calcul des paramètres s'appuie sur des nomogrammes simples, et que le dessin déçoule d'une documentation modèle unifiée. 


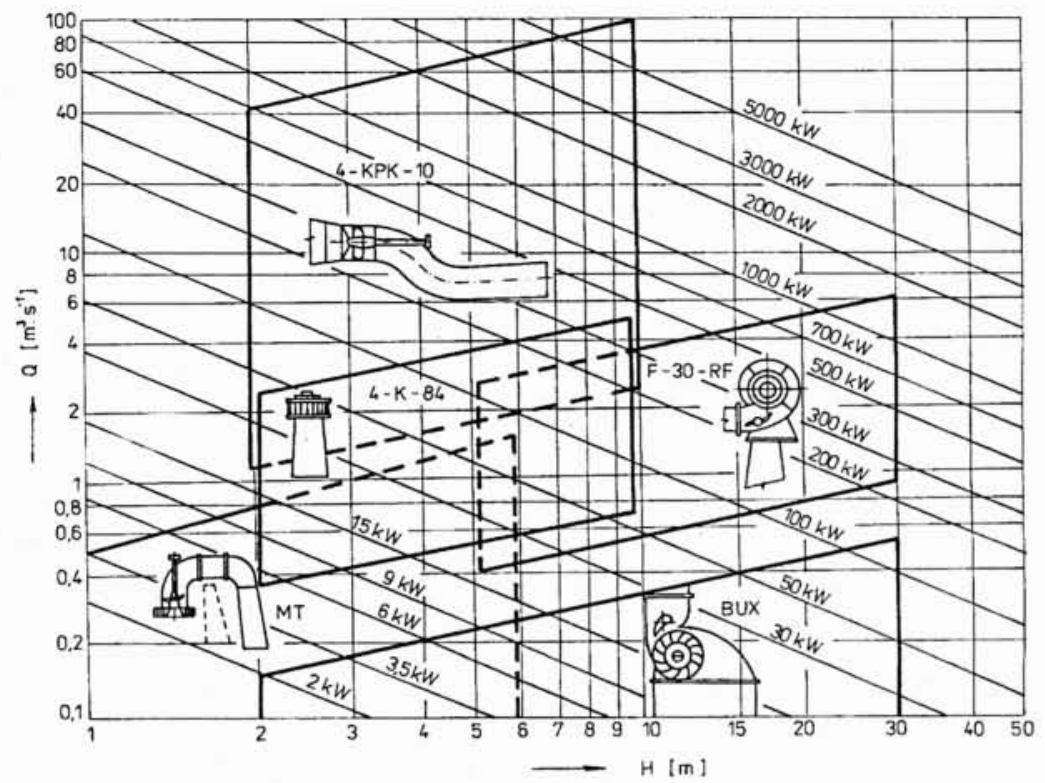

1.

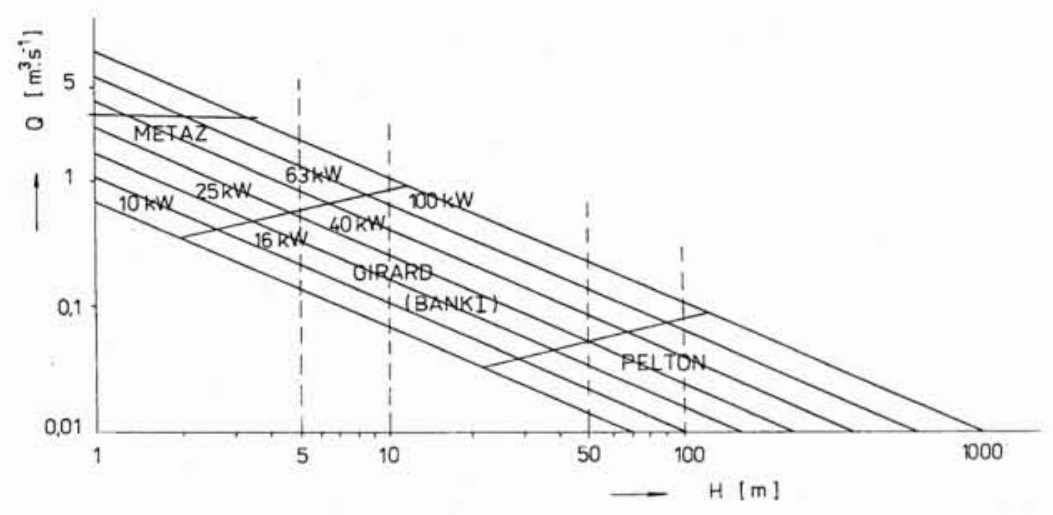

2.

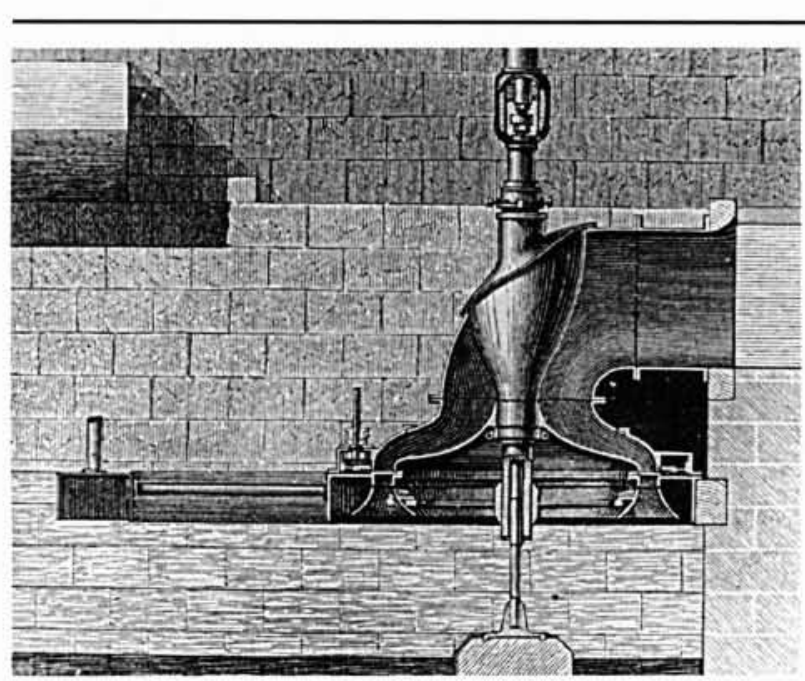

3.

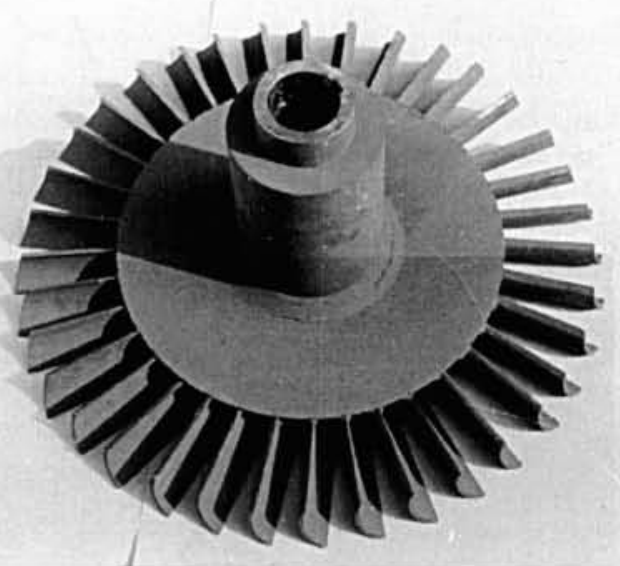

4. 
Les résultats des expériences de l'auteur sont les suivants :

\section{1) Turbine Girard simplifiée}

Les aubes de la roue mobile et de la roue fixe de la turbine Girard d'origine (fig. 3) étaient gauches. Ces roues utilisaient des pièces moulées, les aubes en tôle emboutie étant coulées dans le moyeu et la couronne. L'auteur a remplacé les aubes gauches par des surfaces laminées scellées dans des tubes (fig. 4) ou par des tôles pliées. On a déterminé par le calcul et par la mesure sur modèle que la baisse de rendement reste acceptable.

On a mesuré un rendement global de $70 \%$ sur un modèle de construction artisanale utilisant des aubes de guidage, et de $74 \%$ en utilisant la bâche spirale de Reiffenstein sans aube de guidage. La différence est due à de moindres pertes hydrauliques dans la bâche spirale isobarique de Reiffenstein. Dans le cadre de ces recherches, l'auteur a élaboré une théorie et énoncé mathématiquement les lois de l'écoulement [lors de l'utilisation de la régulation de Reiffenstein] pour la turbine Girard; il a vérifié cette théorie par des mesures sur modèle, les résultats obtenus prouvant le bon accord entre les paramètres calculés et ceux mesurés Q..., t, s.

\section{2) Turbines monodisques à deux étages (Turbines à deux étages sans transmission)}

Elles sont l'objet d'un brevet d'invention de l'auteur. On a disposé sur un seul disque deux étages en série, formant un canal continu, du point de vue hydraulique et du point de vue constructif, à la différence de la turbine Banki, où le canal est interrompu, de sorte qu'il y a un espace sans aubes entre les deux étages; cet espace assure le passage entre le premier et le deuxième étage (fig. 5). L'auteur a fait des recherches théoriques et les a vérifiées sur modèles dans le cas d'une roue mobile à un seul canal (fig. 6) et à plusieurs canaux, jusqu’à 12 (fig. 7). Le rendement hydraulique augmentait avec le nombre de canaux, et atteignait $83 \%$ avec 12 canaux. Dans le cas des solutions à plusieurs canaux, la roue mobile modèle avait été construite de façon artisanale par un ouvrier inexpérimenté dans ce genre de travail, qui a fabriqué en cinq jours une roue à 12 canaux de $400 \mathrm{~mm}$ de diamètre (fig. 7). L'anneau de support [?] et la bague d'étanchéité ont été tournés, les autres travaux exécutés à la main; sur la figure 8 on voit la simplicité du procédé. Le rendement volumétrique était modeste, du fait que le jeu latéral en amont atteignait 1 à $2 \mathrm{~mm}$, ce que l'on peut éviter, même dans une construction artisanale.

Outre sa simplicité de fabrication, ce type de turbine a l'avantage de guider l'eau sur tout son trajet, de l'entrée à la sortie de la roue mobile. C'est pourquoi il n'est pas nécessaire que ce soit une turbine limite comme c'est le cas pour la turbine Banki ; elle peut avoir le caractère d'une turbine de surpression au premier étage d'alimentation. II n'y a pas de pertes dues au changement du diamètre de

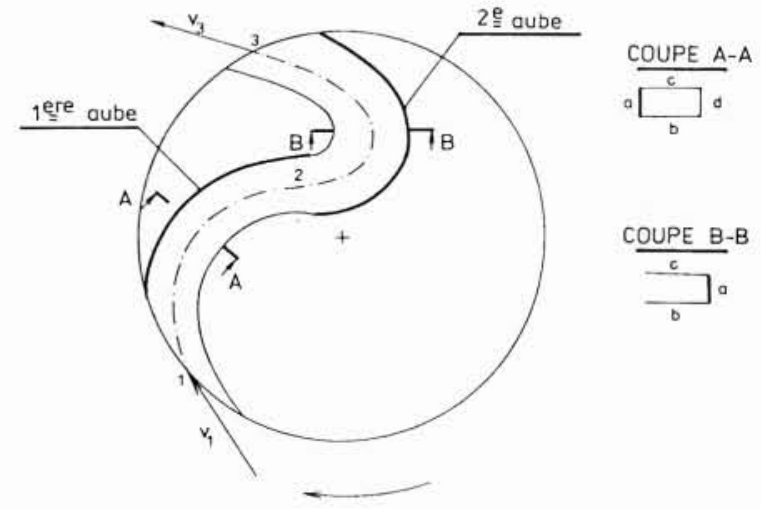

5.

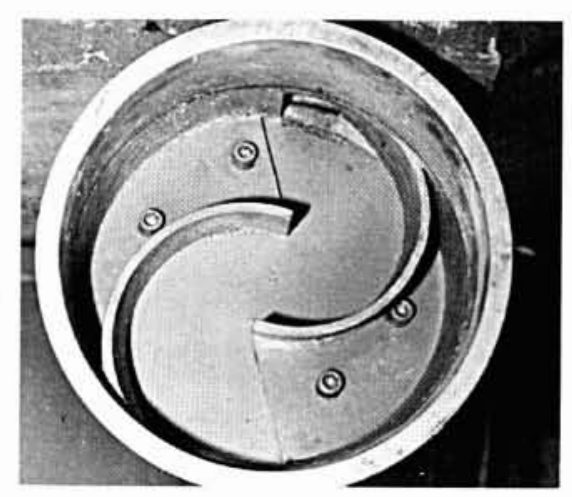

6.
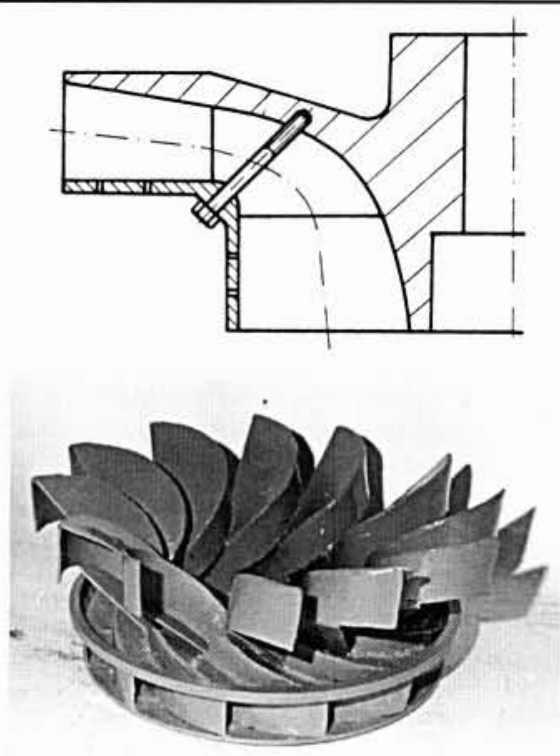

7. 


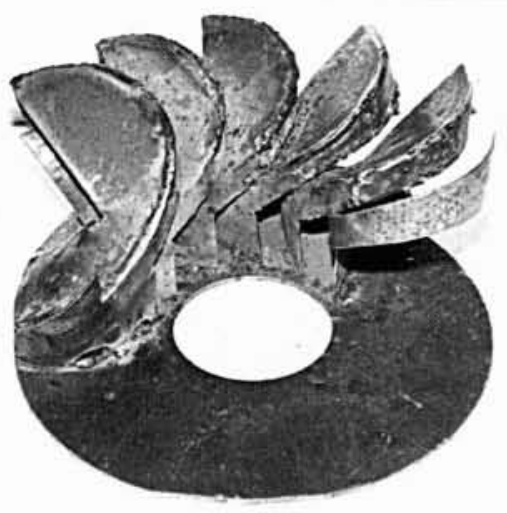

8.
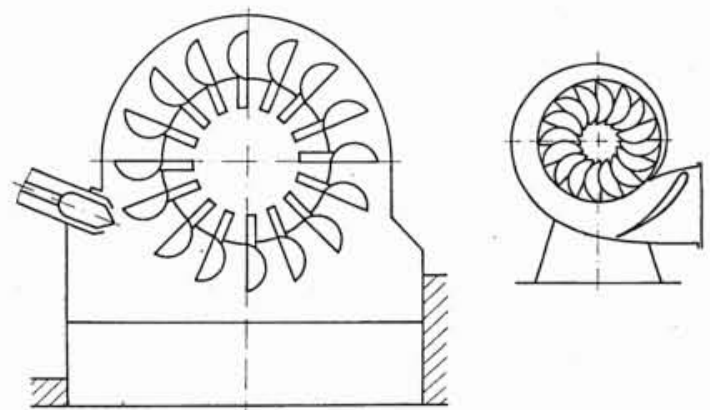

9.

$a_{1}$

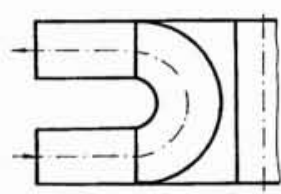

c,

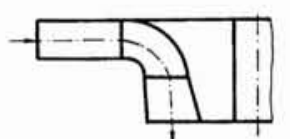

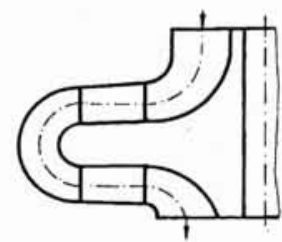

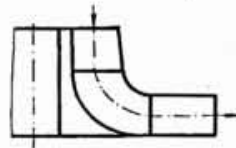

a, Turbine radialo-radiale ovec rove de transmission intérieure

b. Turbine radialo-radinle avec rove de transmission exterieure

c) Turbine radialo-axiale

d, Turbine axialo-radiale

10.
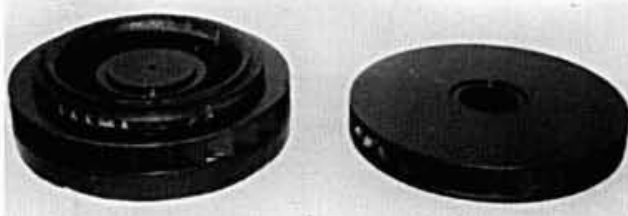

11.

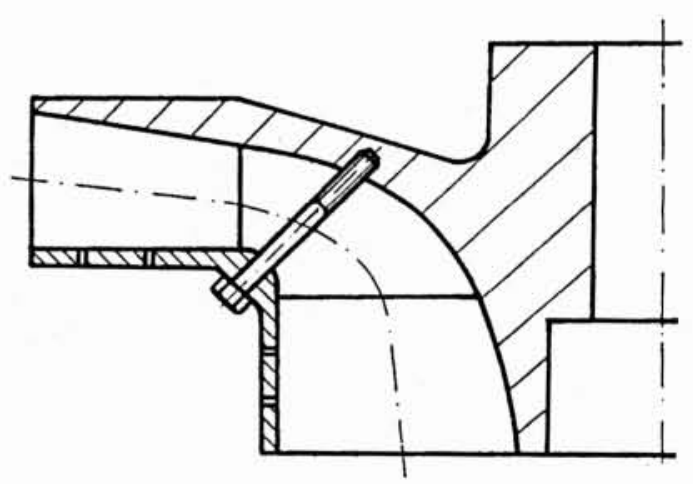

13.

l'écoulement lors du passage d'un étage à l'autre, comme dans la turbine Banki. Par comparaison avec une turbine Pelton de mêmes paramètres $\mathrm{QH}$, les dimensions et le poids sont beaucoup plus faibles (fig. 9).

La simplicité de la fabrication de ce type de turbine résulte de sa simplicité hydraulique. Comme la transformation de l'énergie hydraulique en travail mécanique s'effectue en deux étages, on peut se permettre de simplifier le tracé de l'écoulement dans chacun d'eux, en le rapprochant d'un écoulement bidimensionnel, alors qu'il est tridimensionnel dans la turbine Francis. Les aubes et les parois des canaux n'ont donc plus besoin d'être gauches, et peuvent être réalisées par pliage, sans dispositif spécial ; on satisfait en même temps aux exigences concernant la forme, en accord avec la solution hydraulique optimale. 


\section{3) Turbines bidisques à deux étages}

L'auteur a déduit les turbines bidisques à deux étages des turbines monodisques, afin de simplifier davantage encore l'écoulement dans les canaux de la roue mobile, en répartissant en trois endroits différents le changement de direction et de vitesse. Mis à part l'espacement des aubes, c'est grâce à la roue de transmission sans aubes que l'eau est amenée du premier au deuxième étage de la roue mobile lors du changement de direction. L'auteur l'a fait de quatre façons différentes (fig. 10); la solution est soit une roue mobile radialo-radiale, avec roue de transmission extérieure ou intérieure, soit avec roue mobile radialo-axiale ou une roue axialo-radiale. En répartissant la transformation d'énergie entre trois zones, on a allongé le trajet de l'eau, mais en même temps étendu le choix possible des paramètres de l'écoulement, de façon que les pertes dans la roue mobile ne soient pas plus élevées que dans une turbine Francis. La simplicité de construction découle de celle des formes, et de la possibilité d'effectuer par un système de kits l'assemblage des éléments de la roue mobile. La figure 11 montre une turbine à deux disques avec roue intérieure de transmission démontée. Sur la figure 12 on voit une partie d'une turbine axialo-radiale : il s'agit du moyeu avec aubes soudées. La couronne a été construite séparément et fixée au moyeu par des vis ; on peut aussi utiliser une roue de transmission, comme le montre la figure 13.

Les types de turbines évoqués ci-dessus ont été étudiés théoriquement par l'auteur, qui a vérifié sur modèle la validité des relations théoriques; il a effectué une étude détaillée du rendement global que l'on peut atteindre, et de la baisse admissible de rendement en rapport avec la rentabilité économique des micro-centrales. Ces problèmes ont été résolus en étant en contact direct avec les constructeurs potentiels de tels ouvrages: avec des particuliers, mais aussi avec des entreprises industrielles, en particulier avec les mines, qui ont à leur disposition des groupes de secours rapidement disponibles en cas de panne; ces groupes peuvent être utilisés, en régime d'exploitation normal, à des travaux peu exigeants, par exemple à la construction d'une turbine hydraulique pour les besoins de l'entreprise. L'auteur considère que la question du rendement et de la rentabilité des micro-centrales doit être appréciée différemment du cas des grands ouvrages, où l'on doit satisfaire à des critères techniques et économiques. Dans le cas des micro-centrales déjà construites, leur reconstruction est intéressante, parce qu'elle est assez simple et qu'on peut alors les équiper d'un appareillage plus efficace.

\section{Bibliographie}

[1] Neshleba M., Kopecký V. - Mini and microhydro in Czechoslovakia, Water Power. April 1982.

[2] Hothersall R. - Microhydro turbine selection criteria, Water Power, Februrary 1984. 
Technomic offers Six Courses on Water Quality and Wastewater Treatment

Technomic Publishing Company, Program Division, is offering

the following six water/wastewater treatment courses:

Water Quality and Wastewater Treatment Courses

November 16-17-18, 1993, Stouffer Mayflower Hotel, Washington, D.C.

Program Organizers

W. Wesley Eckenfelder, Jr., Eckenfelder, Inc., U.S.A.

James W. Patterson, Patterson Associates, Inc., U.S.A.

1. Activated Sludge Process Design and Control: Theory and Practice

Topics include: process kinetics, control of sludge quality and filamentous growth, and case histories of industrial and municipal wastewater systems in the U.S. and Europe.

2. Upgrading Wastewater Treatment Plants

Tupics include: stress testing, on-line monitoring,

clarifier testing, dye testing, operations and operator

training, instrumentation and control, and new liquid and solids processing technology.

3. Toxicity Reduction: Evaluation and Control

Topics include: toxicity reduction evaluations,

identification and testing overview; testing procedures;

toxicity reduction methodologies; and case histories.

4. Municipal Sewage Sludge Management

Topics include: planning, designing, and implementing

municipal sewage sludge management projects. Unit operations for processing sludge and available methods for final disposition.

5. Biological Nutrient Removal

Topics include: biological processes for nutrient removal;

modification of existing plants; retrofit case histories;

treatment facilities: design criteria, close operating

parameters, process control, and performance; sludge

handling; and pilot plant studies.

6. Design of Anaerobic Processes for the Treatment of Industrial and Municipal Wastes

Topics include: anaerobic principles, mechanisms and

kinetics; stirred reactors; packed bed reactors;

expanded/fluidized bed systems; upflow anaerobic sludge

blanket (UASB); anaerobic sludge digestion; and case studies:

design criteria, operating parameters and performance.

For detailed course agendas and registration information,

please contact:

Program Division

Technomic Publishing Company, Inc.

851 New Holland Avenue, Box 3535

Lancaster, PA 17604 , U.S.A.

Telephone: 717-291-5609, Fax: 717-295-4538 